


\section{About IJMA [last updated October, $\left.1^{\text {st }}, 2021\right]$}

$\checkmark$ International Journal of Medical Arts is the Official Journal of the Damietta Faculty of Medicine, AlAzhar University, Egypt

$\checkmark$ It is an International, Open Access, Double-blind, Peer-reviewed Journal

$\checkmark$ Published four times a year

$\checkmark$ The First Issue was published in July 2019

$\checkmark$ Published under the following license: Creative Commons Attribution-ShareAlike 4.0 International Public License (CC BY-SA 4.0). It had updated from the Creative Commons license [CC BY] in volume 2, Issue 4, October 2020 About IJMA

$\checkmark$ The Egyptian Knowledge Bank hosts the web site of IJMA

$\checkmark$ The Egyptian Knowledge Bank supports IJMA

$\checkmark$ IJMA follows the regulations of the International Committee of Medical Journal Editors

$\checkmark$ IJMA is indexed in the "Directory of Open Access Journals" [15 January 2021].

$\checkmark$ IJMA is indexed in J-Gate [29-6-2021]

$\checkmark$ IJMA is a member of the International Society of Managing and Technical Editors

$\checkmark$ Listed in "Index Copernicus", "Publons", "Academic resource index [ResearchBib]" "Electronics journal library", "Eurasian Scientific Journal Index", "WorldCat" Superstar Journal Database, and "Citefactor"

$\checkmark$ IJMA introduced to the search engine [BASE] through DOAJ

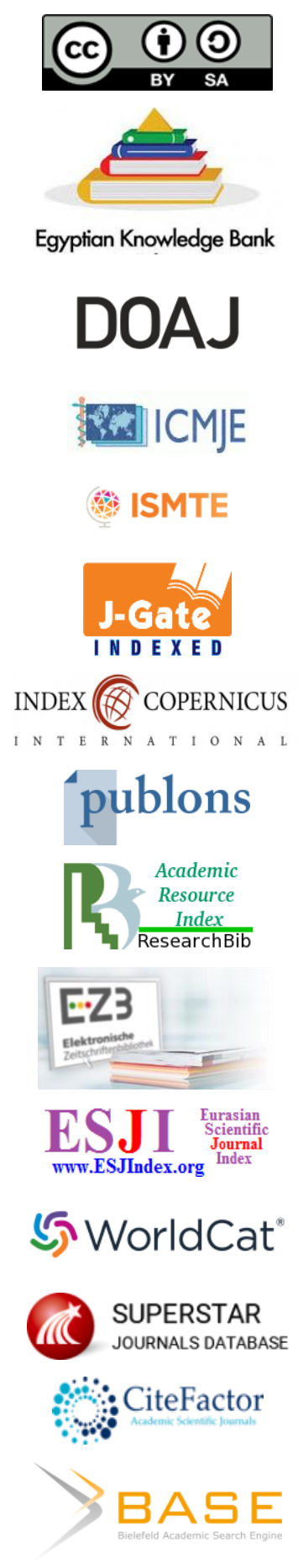




Available online at Journal Website
https://ijma.journals.ekb.eg/
Main subject [Obstetrics and Gynecology]

Original Article

\title{
Prediction of Fetal Lung Maturity by Fetal Pulmonary Artery Doppler in Cases of Severe Pre-Eclampsia
}

\author{
Ashraf Abdelrahim Elkashty [1], Khattab Abdel Halim Omar [1], Samia Mohamad Eid [1]
}

${ }^{1}$ Department of Obstetrics and Gynecology, Damietta Faculty of Medicine, Al-Azhar University, Egypt

Corresponding author: Ashraf Abdelrahim Elkashty

Email: dr.ashraf.alkashty@gmail.com

Submission date: August 11, 2021; Acceptance date: October 6, 2021

DOI: 10.21608/IJMA.2021.90115.1348

DOAJ

\section{ABSTRACT}

Background: Severe pre-eclampsia occurring remote from the term is a decision-making dilemma for the obstetrician, the general recommendation is that women with severe pre-eclampsia should be delivered to avoid maternal complications; others recommend prolonging pregnancy in most cases of severely premature pre-eclamptic gestation until the development of fetal lung maturity, development of fetal or maternal distress, or achievement of gestational age of 34 to 36-week of gestation. The cut-off level of pulmonary artery acceleration time to ejection time ratio $[P A T / E T]$ that determines fetal lung maturity in cases of severe pre-eclampsia $[P E]$ is not determined yet.

Aim of the work: To study the Doppler indices of the main fetal pulmonary artery and their role in predicting respiratory distress syndrome [RDS] in severe pre-eclampsia.

Patients and Methods: A prospective longitudinal cohort research was designed in which 102 pregnant women with PE were chosen and fetal pulmonary artery flow velocity data were acquired by Doppler ultrasound and linked with the development of neonatal RDS.

Results: The AT/ET ratio in the fetal pulmonary artery velocity waveform was found to be directly related to the development of newborn RDS. A cut-off value of 0.3 resulted in a sensitivity of $71.4 \%$, specificity of $79.7 \%$, and total accuracy of $77.5 \%$.

Conclusion: A high AT/ET ratio in the fetal pulmonary artery is related to the future development of RDS in neonates of mothers with severe pre-eclampsia, implying that fetal pulmonary artery Doppler ultrasound may be a valuable tool in the identification of fetal lung maturity in situations of severe pre-eclampsia.

Keywords: Fetal lung maturity; Fetal pulmonary artery; Doppler; Severe pre-eclampsia

This is an open-access article registered under the Creative Commons, ShareAlike 4.0 International license [CC BY-SA 4.0] [https://creativecommons.org/licenses/by-sa/4.0/legalcode.

Citation: Alkashty AA, Omar KA, Eid SM. Prediction of Fetal Lung Maturity by Fetal Pulmonary Artery Doppler in Cases of Severe Pre-Eclampsia. IJMA 2021; 3 [4] OctoberDecember: 1862-1873 [DOI: 10.21608/IJMA.2021.90115.1348]

${ }^{*}$ Main subject and any subcategories have been classified according to the research topic 
Since the advent of ultrasound and antenatal fetal testing, there has been a growing emphasis on optimizing fetal outcomes in difficult, high-risk pregnancies ${ }^{11,2]}$. Preeclampsia affects about $5 \%$ of pregnancies and hypertensive disorders of pregnancy cause over 60,000 maternal fatalities each year around the world [3]. The decision to deliver a woman with severe pre-eclampsia that is far from the term [mid-trimester] is difficult for the obstetrician. The usual suggestion is that women with severe pre-eclampsia be delivered after stabilization to avoid maternal problems ${ }^{[4]}$.

In most cases of highly premature pre-eclamptic gestation, certain institutions propose continuing pregnancy until one of the following occurs development of fetal lung maturity, development of fetal or maternal discomfort, or accomplishment of gestational age of 34 to 36-week of gestation $[5,6]$. Preterm labor [before 37-week] is the most common complication, with seven to ten percent of deliveries resulting in mortality in the second part of pregnancy. One of the primary causes of morbidity and mortality in premature neonates is neonatal respiratory distress syndrome $[R D S]^{[7]}$.

Numerous tests have been developed in an attempt to identify if fetal lung maturation has reached a level sufficient to prevent the development of fetal RDS. These tests are based on four core themes: biochemical testing for active surfactant components, biophysical testing for surfactant functionality, physical testing of amniotic fluid opacity, and ultrasound examination of the fetuses and their tissues $[8,9]$.

Neonatal RDS has been predicted using fetal pulmonary artery Doppler. Kim and colleagues discovered that a high PAT/ET was linked to newborn RDS [10]. The development of RDS in preterm newborns is linked to an increased acceleration time to ejection time [AT/ET] ratio in the fetal pulmonary artery. These findings show that fetal pulmonary artery Doppler velocity could be a reliable noninvasive tool for assessing fetal lung maturity, similar to how middle cerebral artery Doppler has replaced amniocentesis for fetal anemia testing [10].

\section{AIM OF THE WORK}

The cut-off level of PAT/ET that determines fetal lung maturity in cases of severe pre-eclampsia $[\mathrm{PE}]$ is not determined yet. Thus, this work aims to predict fetal lung maturity using the fetal main pulmonary artery Doppler waveform in cases with severe pre-eclampsia and comparing these results with the neonatal outcome to detect the cutoff level of fetal pulmonary artery acceleration time to ejection time ratio [PAT/ET] that determines fetal lung maturity in cases of severe pre-eclampsia [PE].
From October 2016 to September 2019, a prospective longitudinal cohort study was undertaken at the New Damietta Al Azhar University hospital's obstetrics and gynecology department in Egypt. One hundred and two pregnant women with severe pre-eclampsia who received prenatal care in obstetrics' outpatient clinic and gave birth within $24 \mathrm{~h}$ after admission were included in the research.

Inclusion criteria: All pregnant women with severe preeclampsia between 32 and 37- week gestation.

Exclusion criteria: Multiple pregnancies, unclear gestational age, identified fetal congenital defect, preterm labor at gestational age less than 32-week, pregnant women with gestational age more than 37- week, pregnant women who do not meet the criteria for severe pre-eclampsia, multiple pregnancies, and unsure gestational age.

Intervention: According to the definition of the American college of obstetricians and gynecologists 2020, there were 110 patients with severe pre-eclampsia with the following characteristics: thrombocytes 100,000/L, microangiopathic cell lysis LDH, higher serum transaminase levels-ALT or AST, continuous migraine, or other cerebral or visual abnormalities, persistent abdominal discomfort, blood pressure $160 / 110 \mathrm{~mm} \mathrm{Hg}$ and albuminuria $2.0 \mathrm{~g} / 24 \mathrm{~h}$ or $2+$ dipstick and or serum creatinine $>1.2 \mathrm{mg} / \mathrm{dl}$ except if earlier raised.

Methods: for each patient, the following data were obtained:

- Complete medical and surgical history, including obstetric history [preterm labor, stillbirth, IUFD, abortion], current history [to detect symptoms of severe pre-eclampsia such as headache, epigastric discomfort, blurring of vision, and vomiting], family history, and medical and surgical history.

- Clinical examination: blood pressure, pulse, and temperature readings.

- Blood group, fasting, and postprandial blood sugar, thyroid-stimulating hormone, total blood picture to identify platelet count, SGOT, SGPT, LDH, and serum creatinine to diagnose organ affection are fundamental laboratory investigations for pregnant women.

\section{Basic ultrasound assessment}

A single examiner [from the authors' team] conducted all ultrasonic examinations using the Voluson s8 ultrasound machine [GE Healthcare Austria GmbH, Seoul, South Korea] equipped with a 3 to $5 \mathrm{MHz}$ convex array sector transduce 
after a routine ultrasound examination that included fetal biometry, estimated fetal weight, and amniotic fluid index. For $S / D$ ratio determination, the investigator conducted a pulsed wave Doppler assessment of the fetal umbilical artery and middle cerebral artery. In the axial view of the thorax, with the fetuses at rest and no fetal breathing motions, the

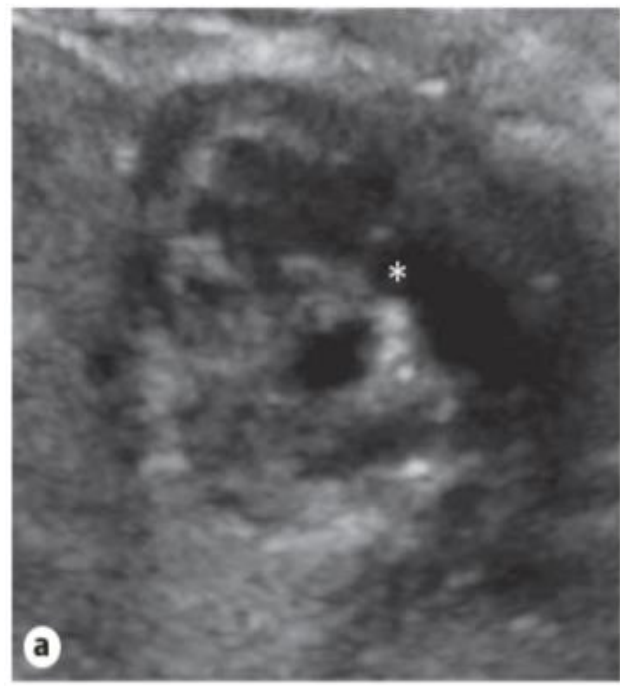

examiner assessed the fetal heart systematically [the fourchamber view, the outflow tracts, and the three-vessel view], then traced the main pulmonary artery [MPA] until midway between the pulmonary valve and the bifurcation of the right and left branches.

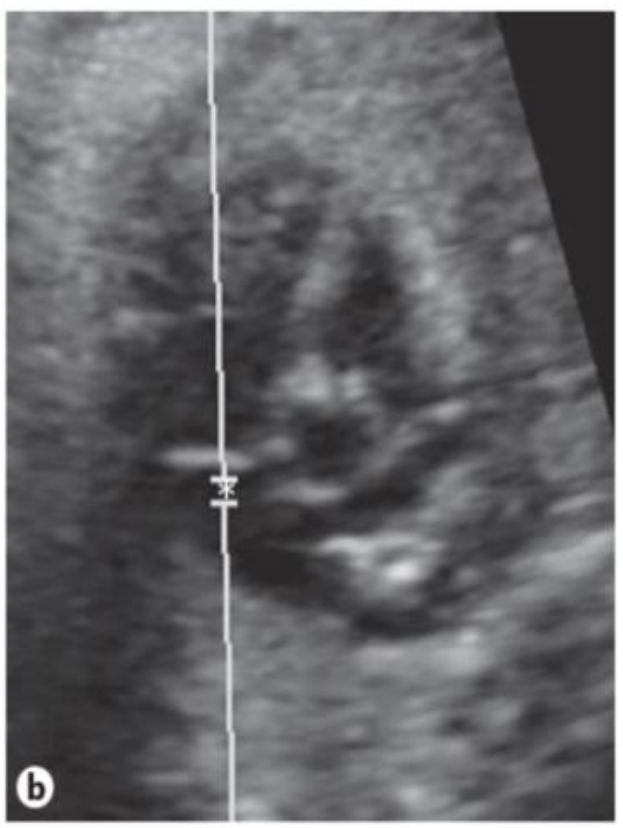

Figure [1]: Views that are good for measuring the PAT/ET. [a] View of the ductal arch. [b] View of the right outflow tract. The area analyzed for obtaining the main pulmonary artery Doppler waveform is indicated by the star.
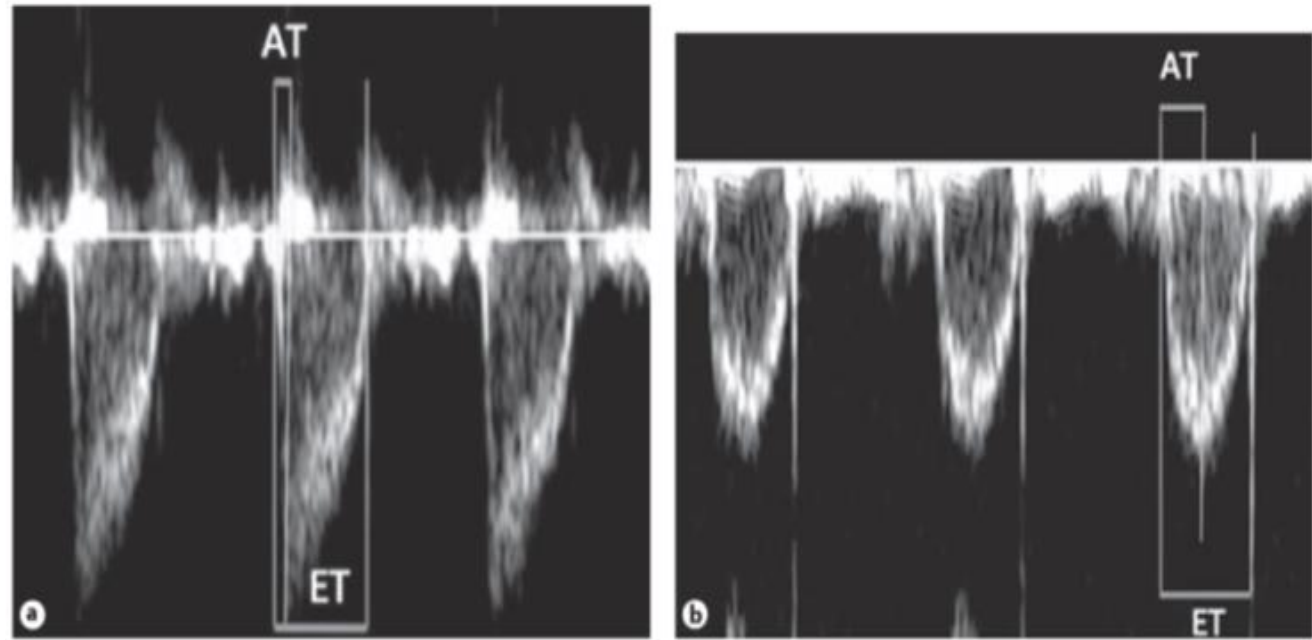

Figure [2]: In the main pulmonary artery Doppler waveform, acceleration [AT] and ejection time [ET] are measured. [a] The wave measurements were taken in an immature state. [b] The measurements were taken on a mature wave

The researchers used a mix of pulsed and color Doppler. The flow waveform of the fetal pulmonary artery [FPAF] was monitored. The FPAF waveform was used to estimate the systolic/diastolic ratio, pulsatility index [PI], resistance index $[R I]$, and acceleration-time/ejection-time ratio [AT/ET] [average values of three waves]. The delay between the onset of ventricular systole and the attainment of peak velocity is known as the [ET]. The lag between the beginning and finish of ventricular systole is known as the [ET].

\section{Neonatal assessment and outcomes}

Neonatal resuscitation and evaluation by a senior neonatologist based on Apgar scores at 5 and $10 \mathrm{~min}$, neonatal ICU entrance, and RDS development as follows: 
[European Consensus Guidelines] Early after delivery, there was evidence of respiratory impairment [tachypnea, retractions, and/or nasal flaring] and sustained oxygen demand for more than $24 \mathrm{~h}$. External pulmonary surfactant infusion, hyaline membrane disorders are seen on radiographs. Comparing those who developed RDS with the normal neonates in the study group, and correlative analysis between fetal main pulmonary artery Doppler findings in two gropes to detect the cut-off level of PAT/ET that determines fetal lung maturity in cases of severe pre-eclampsia.

\section{Study flow}

More than 1500 pregnant woman attending obstetrics outpatient clinic were assessed to predict patient with high risk to develop pre-eclampsia with pregnancy, 277 cases were high risk to develop hypertensive disorders with pregnancy followed up with obstetrics outpatient clinic; 110 cases developed severe pre-eclampsia, 108 cases approved to be part of this study were counseled about the steps of this study and signed on consent including that, and 6 cases were excluded,2 cases delivered earlier than 32 weeks gestation and 4 cases had stillbirth.

The study included one hundred and two pregnant women with severe pre-eclampsia between 32 weeks and 37 weeks gestation were followed up till delivery with demographic, medical and obstetric history, laboratory findings, symptoms of severity, 2D ultrasound and Doppler findings including fetal pulmonary artery Doppler, dexamethasone doses, mode of delivery and gestational age at termination and Neonatal outcome after delivery then correlate between pulmonary artery Doppler findings and development of neonatal RDS [Figure 3].

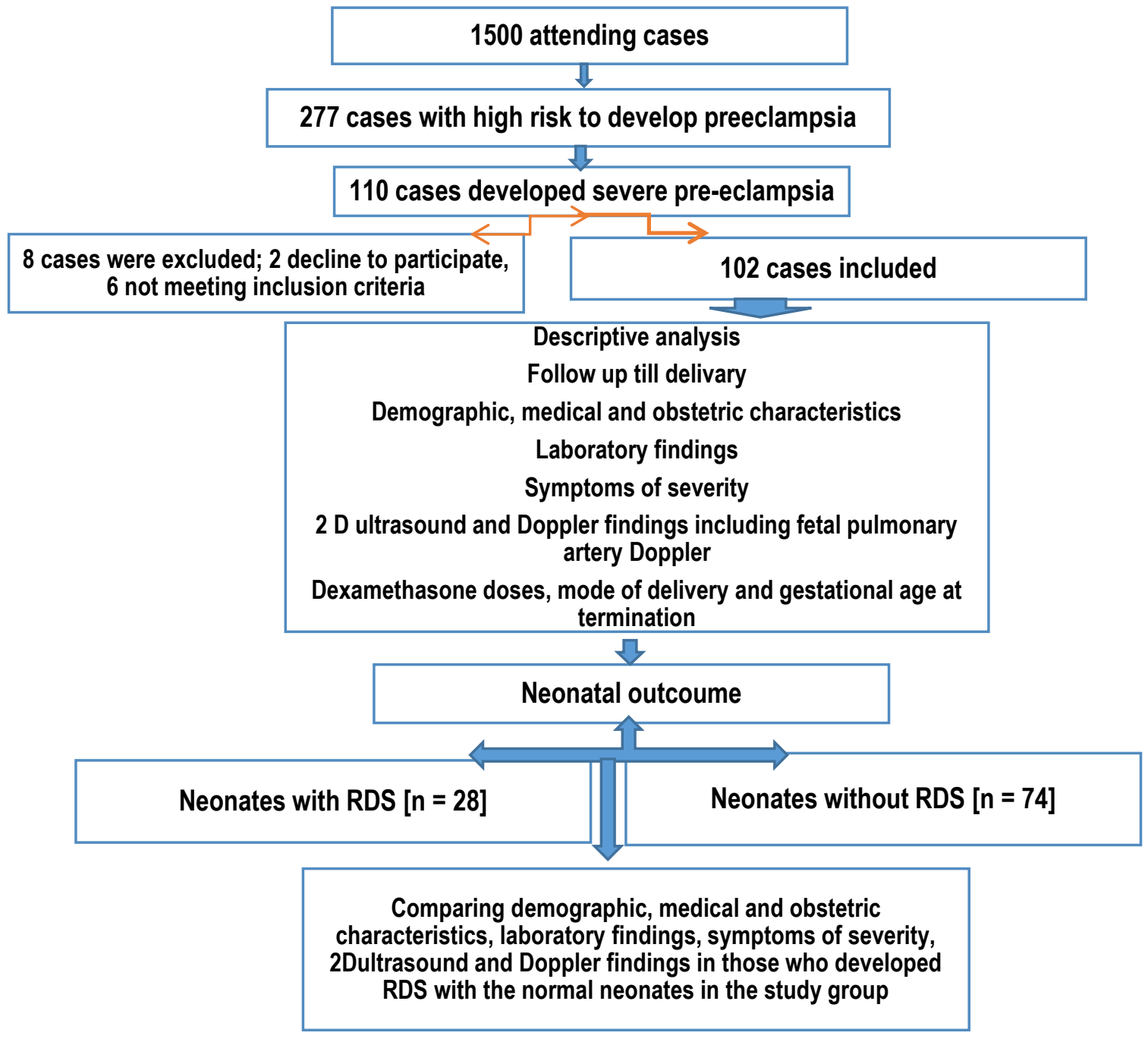

Figure [3]: Flow chart showing study design 
Ethical considerations: The purpose of this study and the role of pulmonary artery Doppler in predicting fetal lung maturity and preterm complications were explained to these patients. One hundred eight cases were approved to participate in this study and were counseled about the procedures of the study and signed informed permission, with 6 instances being disqualified for failing to meet the inclusion criteria. After discussing the research's goal with each patient, the patient must give informed written consent that has been approved by the department's ethical committee [IRB00012367-16-01-006].

Statistical analysis: Data were fed to the computer and analyzed using IBM SPSS software package version 22.0. Qualitative data were described using numbers and percentages. Quantitative data were described using median [minimum and maximum] or non-parametric data and mean, the standard deviation for parametric data after testing normality using the Kolmogorov-Smirnov test. The significance of the obtained results was judged at the [0.05] level. Chi-square test for the comparison of 2 or more groups, Fischer Exact test was used as a correction for the chi-Square test when more than $25 \%$ of cells have a count less than 5 in $2 \times 2$ tables. Quantitative data from two groups were included. The Student t-test, which was utilized, is a type of parametric test that makes assumptions about the parameters of the population distribution from which the sample is derived. This is frequently the assumption that the population data are normally distributed, and non-parametric tests such as the Mann-Whitney and $U$ test, which was used to compare two independent groups, were used to test this assumption. Binary stepwise logistic regression analysis was used for the prediction of independent variables of a binary outcome. Significant predictors in the univariate analysis were entered into the regression model using the forward Wald method/ Enter. Adjusted odds ratios and their 95\% confidence interval were calculated.

Diagnostic accuracy: Receiver Operating Characteristic [ROC] curve analysis: The diagnostic performance of a test or the accuracy of a test to discriminate diseased cases from non-diseased cases is evaluated using Receiver Operating Characteristic [ROC] curve analysis. Sensitivity and Specificity were detected from the curve and PPV, and NPV and accuracy were calculated through cross-tabulation.

\section{RESULTS}

\section{Descriptive part}

Demographic data among studied females: The mean age is 28.57 years with median gravidity \& parity 3 and 2 , respectively. The mean gestational age was 36.09 -week.
Laboratory findings and symptoms of severe preeclampsia among studied females: $50.5 \%$ of the studied cases have proteinuria [+3] and only $3.1 \%$ have proteinuria [+4]. Mean SGOT, SGPT, Serum creatinine, and platelet count are $37.57 \mathrm{u} / \mathrm{l}, 37.57 \mathrm{u} / \mathrm{l}, 0.839 \mathrm{mg} / \mathrm{dl}, \& 171.39 \times 1000$ with median LDH is $487 \mathrm{u} / \mathrm{l}$ and demonstrate that the most frequent symptom was headache [85.3\%], followed by vomiting [41.2\%], epigastric pain [37.3\%] and least was blurring of vision [33.3\%].

Ultrasound and Doppler data among studied cases: mean BPD was [35.3], mean femur length [35.33] and mean amniotic fluid index [10.23], median IUGR was 8, median PAT was 0.064 , median PET was 0.40 , and PAT/ET was 3.75. The mean umbilical artery $S / D$ ratio was 3.04 and the M.C.S/D ratio was 4.09 .

Mode of delivery and gestational age at the termination: $69.6 \%$ of the cases under study delivered through the CS section with mean gestational age were 36.09-week.

Neonatal Data: Regarding neonatal data, $13.7 \%$ of them have respiratory distress syndrome and the same percentage was transient tachypnea of the newborn [TTN]. The mean weight at delivery was 2625 grams. Among study neonates, about $10 \%$ need NICU admission, with a mean duration of stay was 6.53 days. Fetal mortality constituted $2.9 \%$ of neonates.

\section{Analytic part}

Comparing demographic characters and obstetric history of the studied females in those who developed RDS with the normal neonates in the study group, there is no statistically significant association between the incidence of RDS among neonates and age, gravidity, and parity of their mothers. Mean gestational age was significantly lower among neonates with respiratory distress syndrome than without RDS [35.61 versus 36.28 weeks] [Table 1].

Comparing the medical history of the studied females in those who developed RDS with the normal neonates in the study group, there is no statistically significant association between RDS incidence among neonates and the presence of hypertension, diabetes, heart disease, or other medical conditions found among their mothers. Comparing between laboratory findings and symptoms of the studied females in those who developed RDS with the normal neonates in the study group, there is no statistically significant association between neonates with \& without RDS and laboratory results of their mothers including [SGT, SGPT, Presence of proteinuria, serum creatinine and platelet count]. And there is no statistically significant association between the 
presence of new severe persistent headache, projectile resistant vomiting, and persistent blurring of vision among studied mothers and the presence of respiratory distress syndrome among their neonates. However, a higher frequency of epigastric pain was associated with a higher prevalence of RDS [57.1\%] versus $29.7 \%$ of neonates without RDS. Comparing the number of dexamethasone courses in those who developed RDS with the normal neonates in the study group, there is a statistically significant association between dexamethasone administration to pregnant mothers and incidence of respiratory distress syndrome among studied neonates with $85.7 \%$ of cases with respiratory distress have one dose of dexamethasone and $14.3 \%$ only with two doses of dexamethasone [higher dose associated with lower incidence of RDS]. Comparing 2D ultrasound findings in those who developed RDS with the normal neonates in the study group, we found that there is statistically significant higher mean BPD, FL \& AFI among neonates without RDS than those with RDS [35.59 \pm 1.11 , $35.58 \pm 1.30 \& 10.61 \pm 2.67]$ versus [34.54 $\pm 1.55,34.68 \pm 1.83$ $\& 9.21 \pm 2.92$ ], respectively [Table 2].
Comparing Doppler findings in those who developed RDS with the normal neonates in the study group, we found that PET, PAT/ET, and P.S/D ratio were significantly associated with respiratory distress syndrome among studied neonates with P.ET \& P.S/D ratio were higher among neonates with respiratory distress syndrome than neonates without RDS, however, P.AT/ET was higher among neonates with respiratory distress syndrome. Median P.AT, P.RI, P.PI, umbilical artery S/D ratio \& M.C. S/D ratio were not associated with RDS [p>0.05] [Table 3].

Comparing those who developed RDS with the normal neonates in the study group, we found that IUGR, Doppler. PET, Doppler P.S/D ratio \& one cycle of dexamethasone course to mothers were significant predictors of respiratory distress syndrome among study neonates, with $79.5 \%$ of respiratory distress can be predicted by these 4 factors [Table 4]. ROC curve for PAT/ET in differentiating respiratory distress syndrome among study neonates with the area under the curve was excellent and the best-detected cut-off point was 0.3 yielding sensitivity $71.4 \%$ and specificity of $79.7 \%$ with total accuracy was $77.5 \%$ [Table 5$]$.

Table [1]: Association between demographic characters and obstetric history of the females being studied and Respiratory distress syndrome incidence among their neonates

\begin{tabular}{lcccc}
\hline \multicolumn{1}{c}{ Variables } & RDS [-VE] $n=74$ & RDS [+VE] $n=28$ & Test & P \\
\hline Age/years [Mean \pm SD] & $28.79 \pm 5.72$ & $27.96 \pm 6.12$ & $\mathrm{t}=0.64$ & 0.52 \\
Gravidity Median [range] & $3.0[1.0-6.0]$ & $3.0[1.0-5.0]$ & $\mathrm{z}=1.06$ & 0.29 \\
Parity Median [range] & $2.0[0.0-4.0]$ & $2.0[0.0-3.0]$ & $\mathrm{z}=0.59$ & 0.55 \\
Gestational age/weeks [Mean \pm SD] & $36.28 \pm 0.79$ & $35.61 \pm 1.26$ & $\mathrm{t}=3.3$ & $0.002^{*}$ \\
\hline
\end{tabular}

t: Student t-test Z: Mann Whitney U test ${ }^{*}$ statistically significant $[p<0.05]$

Table [2]: Association between 2D Ultrasound findings and respiratory distress syndrome among neonates under study

\begin{tabular}{|c|c|c|c|c|}
\hline 2D US & RDS [-VE] n=74 & RDS [+VE] n=28 & Test & $\mathbf{P}$ \\
\hline BPD Mean \pm SD & $35.59 \pm 1.11$ & $34.54 \pm 1.55$ & $t=3.84$ & $<0.001^{*}$ \\
\hline FL Mean \pm SD & $35.58 \pm 1.30$ & $34.68 \pm 1.83$ & $t=2.78$ & $0.006^{*}$ \\
\hline AFI Mean \pm SD & $10.61 \pm 2.67$ & $9.21 \pm 2.92$ & $t=2.29$ & $0.02^{*}$ \\
\hline IUGR Median [range] & $6[1-9]$ & $8[1-14]$ & $z=2.11$ & $0.03^{*}$ \\
\hline
\end{tabular}

t: Student t-test; * statistically significant z: Mann Whitney U test; BPD: Biparietal diameter, FL: femur length, AFI: amniotic fluid index, IUGR: intrauterine growth retardation

Table [3]: Association between Doppler findings and incidence of respiratory distress syndrome among neonates under study

\begin{tabular}{ccccc} 
Doppler & $\begin{array}{c}\text { RDS [-VE] } \\
\mathbf{n}=74\end{array}$ & $\begin{array}{c}\mathrm{RDS}[+\mathrm{VE}] \\
\mathbf{n}=\mathbf{2 8}\end{array}$ & $\begin{array}{c}\text { Test of } \\
\text { significance }\end{array}$ & $\mathbf{P}$ \\
PAT & $0.058[0.0-0.76]$ & $0.076[0.04-0.23]$ & $\mathrm{z}=0.81$ & 0.42 \\
PET & $0.413[0.04-0.50]$ & $0.347[0.02-0.52]$ & $\mathrm{z}=2.9$ & $\mathbf{0 . 0 0 3}^{*}$ \\
PAT/ET & $0.155[0.0-1.73]$ & $0.41[0.12-7.13]$ & $\mathrm{z}=4.92$ & $<\mathbf{0 . 0 0 1}^{*}$ \\
P.SD. ratio & $6.79[2.28-12.70]$ & $6.40[1.26-10.5]$ & $\mathrm{z}=2.24$ & $\mathbf{0 . 0 2 5}^{*}$ \\
P.RI & $0.85[0.09-1.27]$ & $0.85[0.60-8.7]$ & $\mathrm{z}=0.18$ & 0.86 \\
P.PI & $2.35[1.57-4.7]$ & $2.37[1.57-4.7]$ & $\mathrm{z}=0.19$ & 0.84 \\
Umbilical artery S/D ratio & $2.8[2.3-4.8]$ & $3.01[1.70-5.20]$ & $\mathrm{z}=0.96$ & 0.34 \\
M.C. S/D ratio & $3.80[3.10-6.10]$ & $4.40[2.56-6.8]$ & $\mathrm{z}=1.83$ & 0.07 \\
\hline
\end{tabular}

z: Mann-Whitney $U$ test * statistically significant, all parameters described as median [min-max]

PAT: pulmonary artery acceleration time, PET: pulmonary artery ejection time, PAT/ET: pulmonary acceleration time to ejection time ratio, P.RI: pulmonary resistance index, P.PI: pulmonary pulsatility index, M.C.S/D ratio: middle cerebral systolic to diastolic ratio. 


\begin{tabular}{|l|c|c|c|c|c|}
\hline \multicolumn{1}{|c|}{ Predictor[s] } & B & P value & Odds ratio & \multicolumn{2}{c|}{$\mathbf{9 5 . 0 \% \text { C.I. for odds ratio }}$} \\
\hline Gestational age weeks & & & & Lower & Upper \\
\hline IUGR & -.888 & .213 & .412 & .102 & 1.662 \\
\hline Epigastric pain [-ve] & 0.25 & $0.04^{*}$ & 1.24 & 1.10 & 7.85 \\
\hline BPD.2D.US & 1.172 & .077 & 3.228 & .881 & 11.826 \\
\hline FL.2D.US & -.693 & .198 & .500 & .174 & 1.438 \\
\hline AFI.2D.US & .437 & .264 & 1.549 & .719 & 3.336 \\
\hline Doppler P.ET & -.074 & .578 & .928 & .714 & 1.207 \\
\hline Doppler P. S/D ratio & -10.234 & $.002^{*}$ & .000 & .000 & .027 \\
\hline Dexamethasone course [one & -.455 & $.007^{*}$ & .634 & .456 & .883 \\
\hline cycle] & 5.473 & $.001^{*}$ & 238.060 & 8.323 & 680.9 \\
\hline Constant= & & & & \\
\hline
\end{tabular}

Cl: Confidence interval

Table [5]: Validity of PAT/ET in differentiating RDS among neonates under study

\begin{tabular}{|c|c|c|c|c|c|c|c|}
\hline & $\begin{array}{c}\text { AUC } \\
{[95 \% \mathrm{Cl}]}\end{array}$ & Cut-off point & Sensitivity [\%] & Specificity [\%] & PPV [\%] & NPV [\%] & $\begin{array}{c}\text { Accuracy } \\
{[\%]}\end{array}$ \\
\hline P.AT/ET & $\begin{array}{c}0.816 \\
{[0.73-0.91]}\end{array}$ & 0.30 & 71.4 & 79.7 & 57.1 & 88.1 & 77.5 \\
\hline
\end{tabular}

AUC: area under the curve, PPV: positive predictive value, NPV: negative predictive value

\section{ROC Curve of PATIET in differentiating cases with RDS}

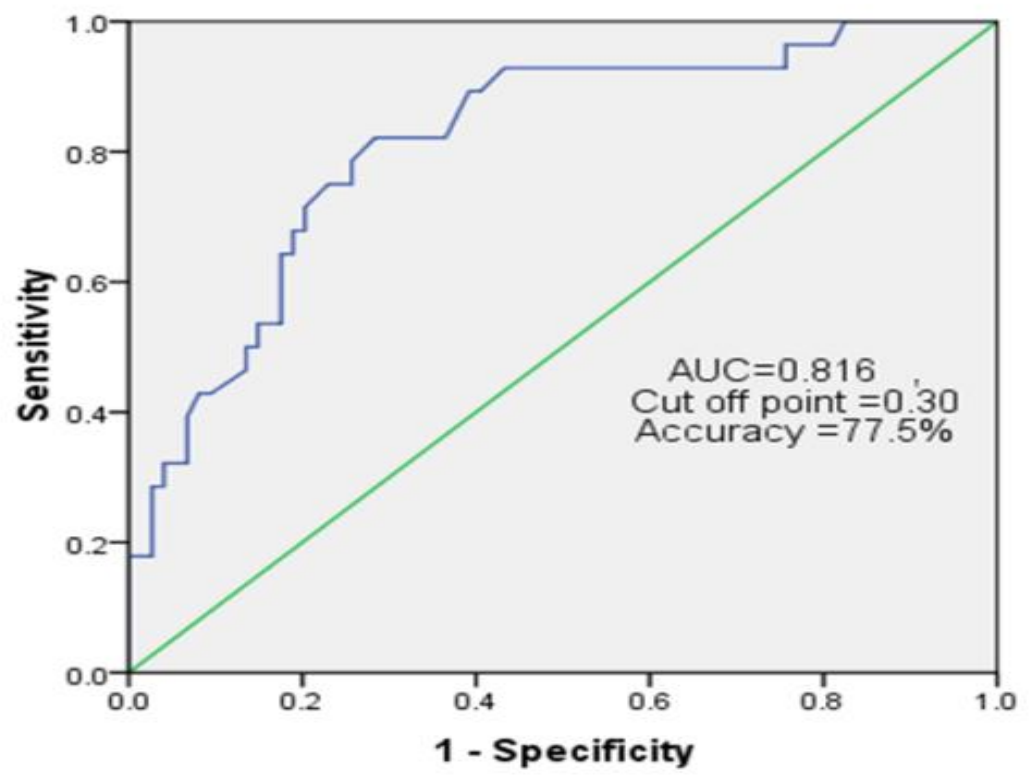

Diagonal segments are produced by ties.

Figure [1]: ROC curve of PAT/ET for differentiating cases with RDS. 


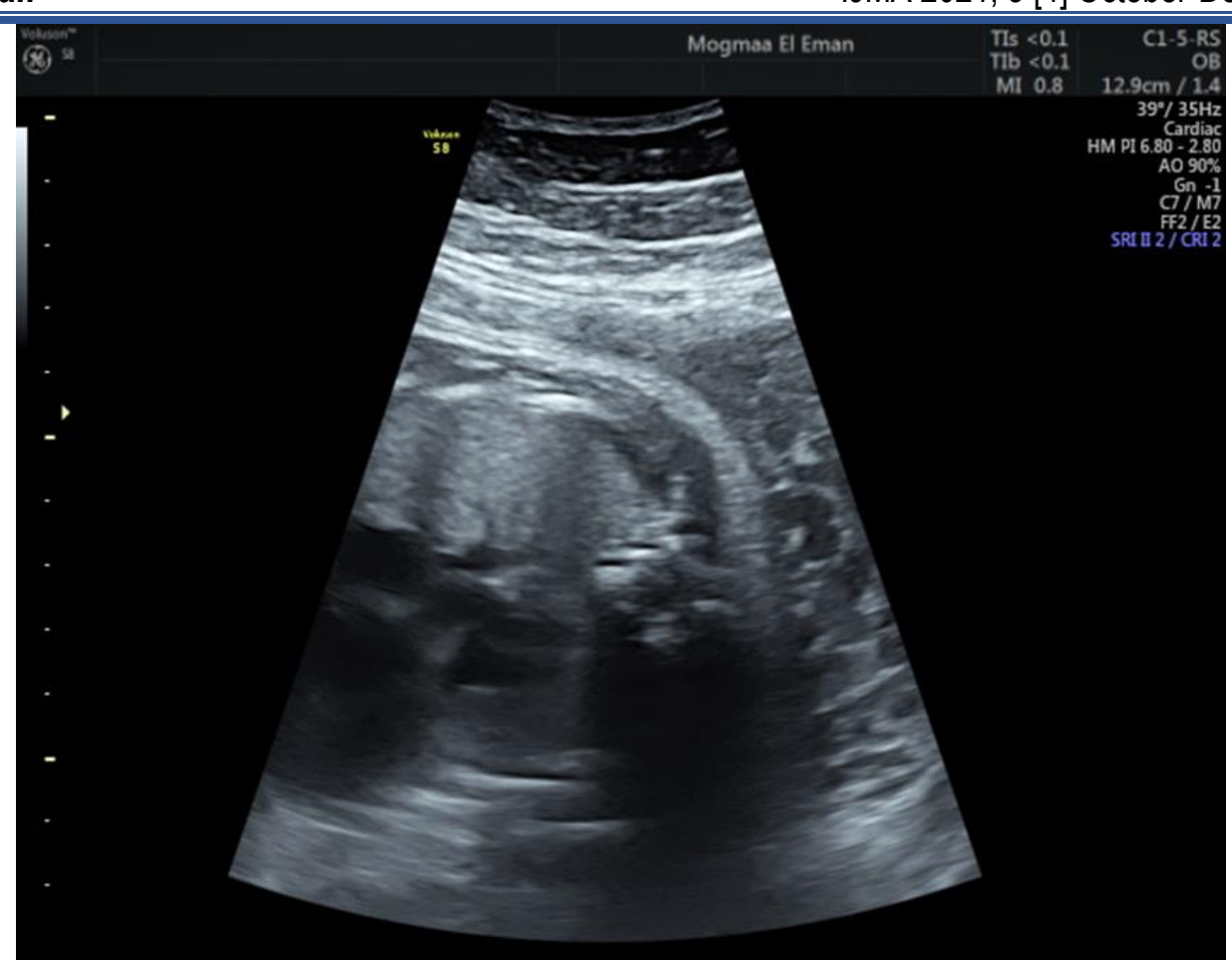

Figure [5]: fetal echo with right ventricular outflow tract view.

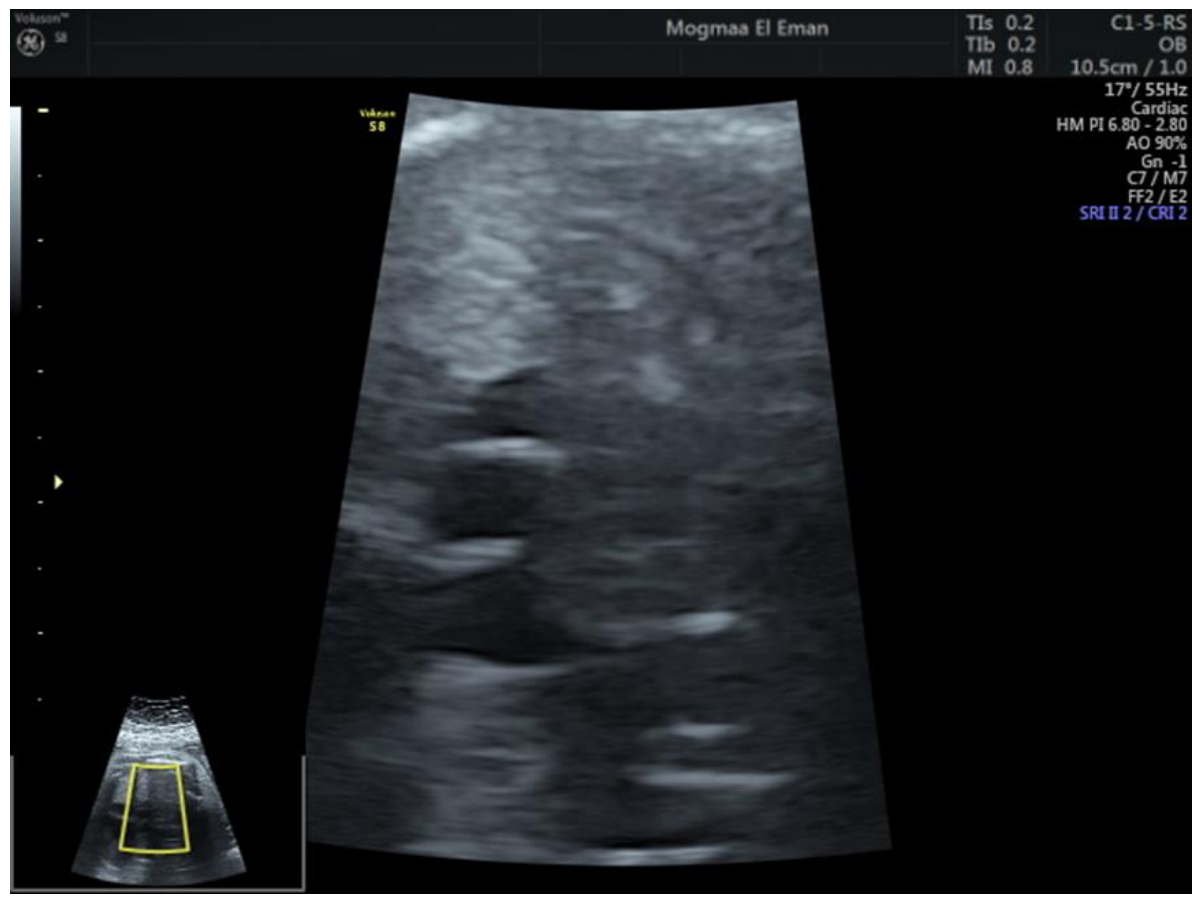

Figure [6]: fetal echo with 3 vessels view. 


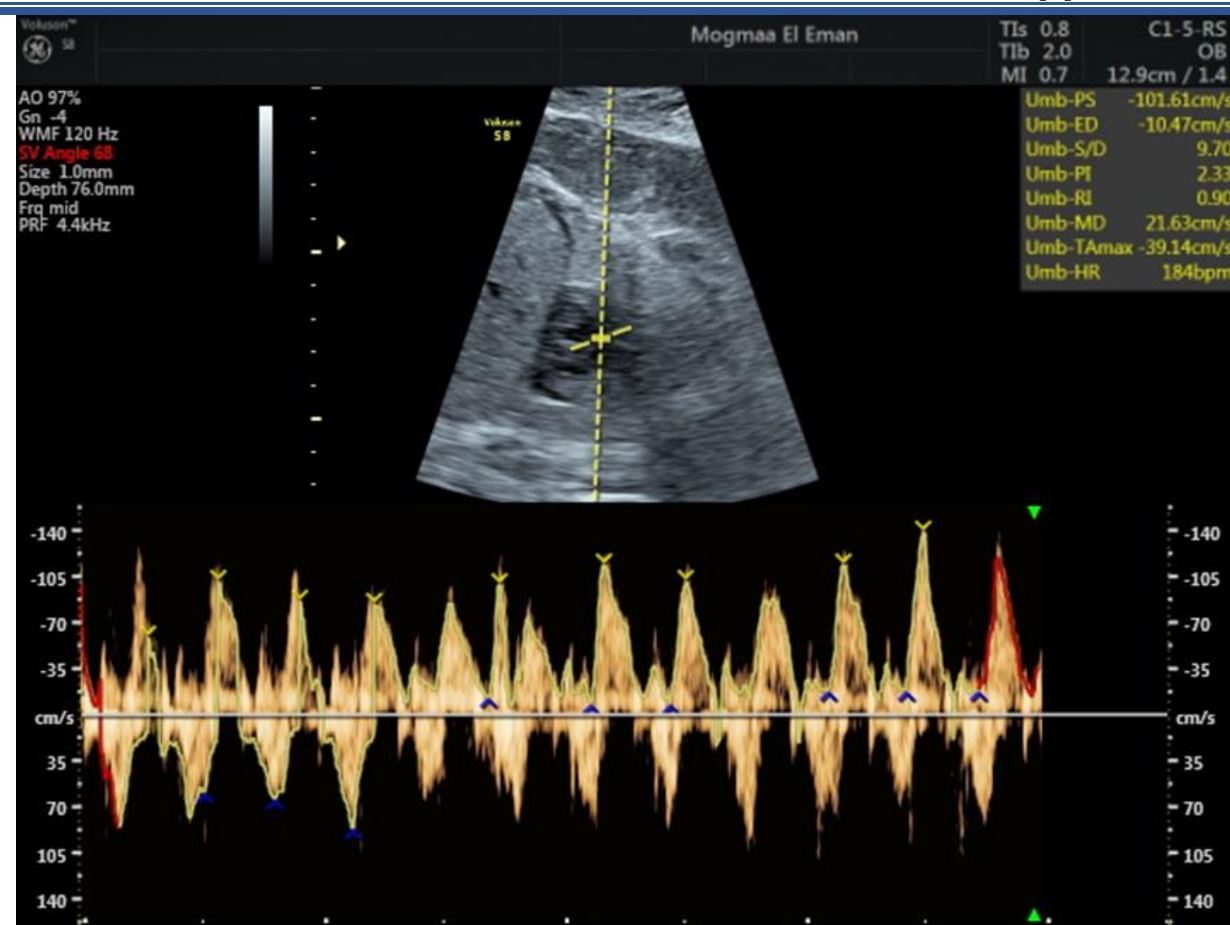

Figure [7]: fetal pulmonary artery Doppler.

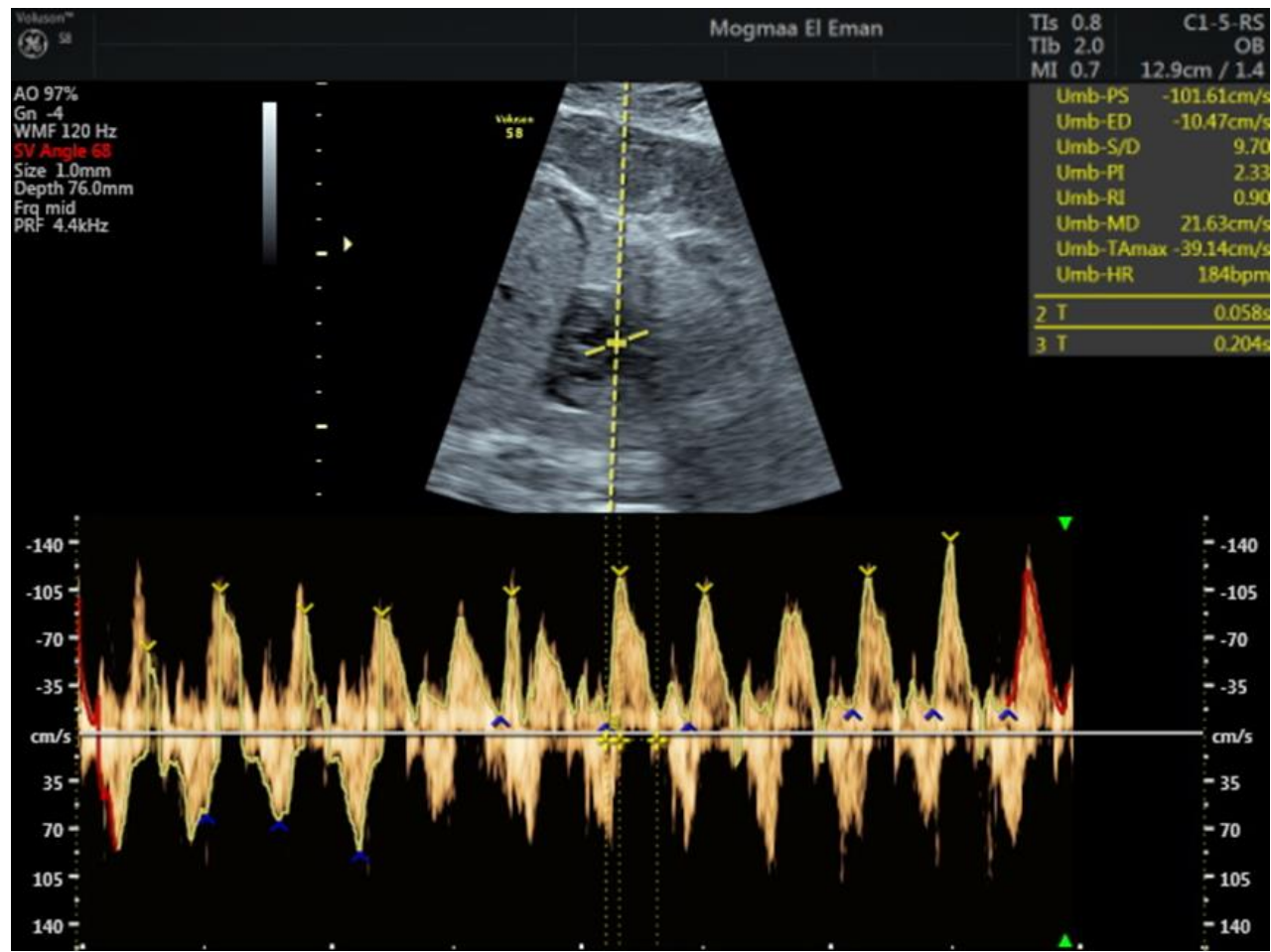

Figure [8]: fetal pulmonary artery AT/ET.

\section{DISCUSSION}

Results of the current work revealed that, there was no statistical significance between the collected demographic data [maternal age, gravidity, and parity] and RDS in cases of severe pre-eclampsia despite gestational age at the time of termination which has a statistical significance with the development of fetal RDS in cases of severe pre-eclampsia. These findings go with the result of a large study on 13,490 VLBW infants, including 2,200 [16.3\%] cases born to mothers with pre-eclampsia in Taiwan which concluded that GA, but not birth weight, was associated negatively with RDS. GA and birth weight were associated inversely with severe RDS, with an OR of $0.68[95 \% \mathrm{Cl}, 0.65-0.7]$ and 0.94 
[95\% Cl, 0.91-0.97], respectively [11].

In this study, there was no statistical significance between medical history in the studied cases and the development of RDS in delivered infants; these results agree with the results cited by Hochberg et al. [12] that support the link between pre-eclampsia in moms and preterm newborns with broncho-pulmonary dysplasia suggesting that chronic hypertension is protective for preterm neonates [13]. In contrast to our study, Li et al. ${ }^{[14]}$ concluded that maternal diabetes, including gestational diabetes mellitus and pregestational diabetes mellitus is connected to a higher incidence of neonatal RDS. Another study found that the risk of pre-eclampsia increased with the severity of diabetes in women with pre-gestational diabetes mellitus. Proteinuria early in pregnancy was linked to a significant increase in negative neonatal outcomes, even when pre-eclampsia was not present [15]. These difficulties are thought to emerge in DM because surfactant synthesis is delayed as a result of maternal hyperglycemia ${ }^{[16]}$, and reduced fluid clearance in the fetal lungs ${ }^{[17]}$. The controversy between these results could be explained as in our study severe pre-eclampsia stimulate early surfactant synthesis and the patients gave good glycemic control.

In this study, there was no statistical significance between laboratory findings and RDS among cases being studied, this finding goes with a large systematic quantitative review [18] regarding proteinuria, and coincide with many other studies] regarding liver function tests ${ }^{[19-21]}$.

In this study, we found no statistically significant relationship between symptoms of severe pre-eclampsia and RDS among study cases despite epigastric pain, these findings correlated with findings of a large study on 13,490 patients by Yu-Hua Wen [11].

In this study, there was a statistically significant inverse relationship between $2 \mathrm{D}$ Ultrasound findings as regard BPD, $F L, A F I$ and IUGR, and RDS among the cases being studied. These results agreed with Gilbert and Danielsen [22] who cited that prematurity linked to poor neonatal outcomes [RDS, IVH, NEC, and CHA] was significantly influenced by IUGR in the third trimester. These results agreed with the results of Rabinovich et al. [23] Who cited that among women with pre-eclampsia who gave birth prematurely, oligohydramnios is an independent risk factor for perinatal morbidity, and these results go with another study which found that gestational age at the time of termination is a good predictor for neonatal outcome in cases of severe preeclampsia [11]. A relatively recent study concluded that even in the context of fetal development limitation, the obstetrician should try to extend pregnancies complicated by early-onset severe pre-eclampsia up to 32 gestational weeks as far as maternal conditions allow. This type of management policy could help to enhance newborn outcomes [24].

Regarding fetal pulmonary artery ejection time and acceleration time to ejection time ratio, there was statistically significant relationship between Doppler indices and neonatal RDS among cases examined in this investigation. These findings corresponded with those of a recent study done by Büke et al. ${ }^{[25]}$ who stated that the fetal PAT/ET ratio is a promising noninvasive technique for predicting RDS in preterm births, and the results of another study, which discovered that measuring FLV or PA-RI can predict RDS in preterm fetuses when these metrics were used together, their predictive power increased [26].

In this research, there was a statistically significant inverse relationship between giving one corticosteroid course and neonatal RDS, this finding agrees with the results of another research which concluded that steroids reduce respiratory distress syndrome [27]. In contrast to our result, Witlin et al. [28] found no relationship between corticosteroid and neonatal outcome, and this difference could be explained as in their study they examine patients delivered from 24 to 33- week gestation, and we study cases 32 to 37- week gestation. Furthermore, Crowther et al. [29] found that after the first course of prenatal corticosteroids, mothers at continued risk of preterm birth received repeat doses, which reduced the likelihood of their infant requiring respiratory support after birth and resulted in a neonatal benefit, and the difference between the results could be explained as in our study 6 patients didn't receive any corticosteroid courses with no neonatal RDS as they developed late-onset pre-eclampsia after lung maturity, 91 patient received one course of corticosteroid with a statistically significant reduction in the development of neonatal RDS and 5 patient received two corticosteroid courses with 4 patients developed neonatal RDS as they developed early-onset pre-eclampsia with its adverse effect on neonatal outcome ${ }^{[30]}$.

The development of respiratory distress syndrome [RDS] in the neonates of mothers with severe pre-eclampsia during pregnancy is linked to an elevated acceleration time/ejection time ratio [AT/ET] with a cut-off level of 0.30 in the fetal pulmonary artery, with a sensitivity 71.4 and specificity 79.7 .

These results are compatible with the results of recent research examining 105 patients and they found that even after accounting for gestational age, estimated fetal weight, and fetal gender, researchers discovered an adverse connection between the diagnosis of RDS in neonates and PAT/ET levels [ $r=0.52$ and $p=.0017]$. A cut-off value of 0.327 yielded $77.1 \%$ specificity, $90.9 \%$ sensitivity, $95.4 \%$ negative predictive value and $52.7 \%$ positive predictive value [25]. Another study found that for subsequent diagnosis of 
TTN in small for gestational age [SGA] neonates, the cut-off value of 0.298 offered ideal specificity of $93.0 \%$ and sensitivity of $81.0 \%{ }^{[31]}$.

This study had many strength points; it is the first one that discusses the cut-off level of PAT/ET in the prediction of neonatal RDS in cases of severe pre-eclampsia. Preeclampsia is a dynamic process, so we can terminate the pregnancy once fetal lung maturity is detected and before deterioration of the maternal condition, we can use this parameter in the prediction of neonatal RDS in other highrisk pregnancies as there were many medical disorders were included in this study accompanied with pre-eclampsia as diabetes mellitus and heart disease, and a wide range of maternal age included in this study. We can measure PAT/ET with two D ultrasound with Doppler with no need for a high-end ultrasound machine or 4D ultrasound; we can predict fetal lung maturity with a non-invasive technique instead of other invasive techniques with its drawbacks.

There are some limitations of the study; many cases included at the beginning of this reassure were delivered either before 32-week gestation or after 37-week gestation, so they were excluded from the study, fetal echo performance is difficult at a gestational age between 32 and 37-week gestation because of high echogenicity of fetal ribs, so anterior position of the fetal chest is preferred to get an optimum view, severe pre-eclampsia is associated with oligohydramnios which adds more difficulty in the performance of fetal echo and the detection of fetal pulmonary artery Doppler indices.

\section{CONCLUSION}

The fetal P.ET, P.AT/ET, and P.S/D ratios detected through Doppler findings were significantly associated with respiratory distress syndrome among neonates, with P.ET, P.SD, and P.AT/ET ratios being higher among neonates with respiratory distress syndrome than neonates without RDS. IUGR, Doppler P.ET, Doppler P.S/D ratio \& one cycle of dexamethasone course to mothers were significant predictors of respiratory distress syndrome among neonates under study. The best-detected cut-off point of the P.AT/ET ratio for the detection of neonatal RDS in cases with severe pre-eclampsia between $32 w$ to 37 - week gestation was 0.3 .

Financial and Non-financial Relationships and Activities of Interest

None

\section{REFERENCES}

1. Varner S, Sherman C, Lewis D, Owens S, Bodie F, McCathran $\mathrm{CE}$, Holliday N. Amniocentesis for fetal lung maturity: will it become obsolete? Rev Obstet Gynecol. 2013;6[3-4]:126-34. PMID: 24826202

2. Åhman A, Edvardsson $\mathrm{K}$, Fagerli TA, Darj E, Holmlund S, Small R, Mogren I. A much valued tool that also brings ethical dilemmas - a qualitative study of Norwegian midwives' experiences and views on the role of obstetric ultrasound. BMC Pregnancy Childbirth. 2019 Jan 16;19[1]:33. DOl: 10.1186/s12884-019-2178-x.

3. Duhig K, Vandermolen B, Shennan A. Recent advances in the diagnosis and management of pre-eclampsia. F1000Res. 2018 Feb 28; 7:242. DOI: 10.12688/f1000research.12249.1.

4. Gestational Hypertension and Pre-eclampsia: ACOG Practice Bulletin, Number 222. Obstet Gynecol. 2020 Jun;135[6]:e237e260. DOI: 10.1097/AOG.0000000000003891.

5. Churchill D, Duley L, Thornton JG, Moussa M, Ali HS, Walker $\mathrm{KF}$. Interventionist versus expectant care for severe preeclampsia between 24 and 34 weeks' gestation. Cochrane Database Syst Rev. 2018 Oct 5;10[10]:CD003106. DOI: 10.1002/14651858.CD003106.pub3.

6. Vigil-De Gracia $\mathrm{P}$, Ludmir J. Conservative management of early-onset severe pre-eclampsia: comparison between randomized and observational studies a systematic review. J Matern Fetal Neonatal Med. 2020 Sep 10:1-8. DOl: 10.1080/14767058.2020.1814249.

7. Yismaw $A E$, Tarekegn AA. Proportion and factors of death among preterm neonates admitted in University of Gondar comprehensive specialized hospital neonatal intensive care unit, Northwest Ethiopia. BMC Res Notes. 2018 Dec 6;11[1]:867. DOI: 10.1186/s13104-018-3970-9.

8. Maged A, Youssef G, Hussien A, Gaafar H, Elsherbini M, Elkomy $\mathrm{R}$, et al. The role of three-dimensional ultrasonography fetal lung volume measurement in the prediction of neonatal respiratory function outcome. J Matern Fetal Neonatal Med. 2019;32[4]:660-665. DOI: 10.1080/14767058.2017.1387898.

9. Vahdaninia $M$, Mackenzie $H$, Dean $T$, Helps S. The effectiveness of $\omega-3$ polyunsaturated fatty acid interventions during pregnancy on obesity measures in the offspring: an upto-date systematic review and meta-analysis. Eur J Nutr. 2019 Oct;58[7]:2597-2613. DOI: 10.1007/s00394-018-1824-9.

10. Kim SM, Park JS, Norwitz ER, Hwang EJ, Kang HS, Park CW, Jun JK. Acceleration time-to-ejection time ratio in fetal pulmonary artery predicts the development of neonatal respiratory distress syndrome: a prospective cohort study. Am J Perinatol. 2013 Nov;30[10]:805-12. DOI: 10.1055/s-00321333132

11. Wen YH, Yang HI, Chou HC, Chen CY, Hsieh WS, Tsou KI, Tsao PN; Taiwan Premature Infant Developmental Collaborative Study Group. Association of Maternal Preeclampsia with Neonatal Respiratory Distress Syndrome in Very-Low-Birth-Weight Infants. Sci Rep. 2019 Sep 
13;9[1]:13212. DOI: 10.1038/s41598-019-49561-8.

12. Hochberg A, Pardo A, Oron G, Krispin E, Amikam U, Wiznitzer A, Hadar E, Salman L. Perinatal outcome following induction of labor in patients with good glycemic controlled gestational diabetes: does timing matter? Arch Gynecol Obstet. 2019 Aug;300[2]:299-303. DOI: 10.1007/s00404-019-05183-z.

13. Rocha GM, de Lima FF, Machado AP, Guimarães HA; Hypertensive Disorders of Pregnancy Study Group. Hypertensive Disorders during Pregnancy and Risk of Bronchopulmonary Dysplasia in Very Preterm Infants. Am J Perinatol. 2019 Jan;36[2]:176-183. DOI: 10.1055/s-00381660865

14. Li Y, Wang W, Zhang D. Maternal diabetes mellitus and risk of neonatal respiratory distress syndrome: a meta-analysis. Acta Diabetol. 2019 Jul;56[7]:729-740. DOI: 10.1007/s00592-01901327-4.

15. Sibai BM, Caritis S, Hauth J, Lindheimer M, VanDorsten JP, MacPherson C, et al. Risks of pre-eclampsia and adverse neonatal outcomes among women with pregestational diabetes mellitus. National Institute of Child Health and Human Development Network of Maternal-Fetal Medicine Units. Am J Obstet Gynecol. 2000 Feb;182[2]:364-9. DOI: 10.1016/s00029378[00]70225-0.

16. Gewolb IH, O'Brien J. Surfactant secretion by type ॥ pneumocytes is inhibited by high glucose concentrations. Exp Lung Res. 1997 May-Jun;23[3]:245-55. DOI: 10.3109/ 01902149709087370.

17. Persson B, Hanson U. Neonatal morbidities in gestational diabetes mellitus. Diabetes Care. 1998 Aug;21 Suppl 2:B7984. PMID: 9704232.

18. Thangaratinam S, Coomarasamy A, O'Mahony F, Sharp S, Zamora J, Khan KS, Ismail KM. Estimation of proteinuria as a predictor of complications of pre-eclampsia: a systematic review. BMC Med. 2009 Mar 24;7:10. DOI: 10.1186/17417015-7-10.

19. Thangaratinam S, Koopmans CM, Iyengar S, Zamora J, Ismail KM, Mol BW, Khan KS; TIPPS [Tests in Prediction of Preeclampsia's Severity] Review Group. Accuracy of liver function tests for predicting adverse maternal and fetal outcomes in women with pre-eclampsia: a systematic review. Acta Obstet Gynecol Scand. 2011 Jun;90[6]:574-85. DOI: 10.1111/j.16000412.2011.01112.x.

20. Mol BWJ, Roberts CT, Thangaratinam S, Magee LA, de Groot CJM, Hofmeyr GJ. Pre-eclampsia. Lancet. 2016;387[10022]: 999-1011. DOI: 10.1016/S0140-6736[15]00070-7.

21. Thygesen SK, Olsen M, Østergaard JR, Sørensen HT. Respiratory distress syndrome in moderately late and late preterm infants and risk of cerebral palsy: a population-based cohort study. BMJ Open. 2016 Oct 11;6[10]: e011643. DOI: 10.1136/bmjopen-2016-011643.
22. Gilbert WM, Danielsen B. Pregnancy outcomes associated with intrauterine growth restriction. Am J Obstet Gynecol. 2003 Jun;188[6]:1596-9; discussion 1599-601. DOI: 10.1067/mob. 2003.384 .

23. Rabinovich A, Holtzman K, Shoham-Vardi I, Mazor M, Erez O. Oligohydramnios is an independent risk factor for perinatal morbidity among women with pre-eclampsia who delivered preterm. J Matern Fetal Neonatal Med. 2019 Jun;32[11]:17761782. DOI: $10.1080 / 14767058.2017 .1417377$.

24. Sato Y, Moriuchi K, Sakae-Matsumoto C, Ueda M, Fujita K. Factors contributing to favourable neonatal outcomes in earlyonset severe pre-eclampsia. J Obstet Gynaecol. 2021 Jan;41[1]:60-65. DOI: 10.1080/01443615.2019.1706160.

25. Büke B, Destegül E, Akkaya $H$, Şimşek D, Kazandi $M$. Prediction of neonatal respiratory distress syndrome via pulmonary artery Doppler examination. J Matern Fetal Neonatal Med. 2019 May;32[10]:1640-1645. DOI: 10.1080/14767058.2017.1413549.

26. Laban M, Mansour GM, El-Kotb A, Hassanin A, Laban Z, Saleh $A$. Combined measurement of fetal lung volume and pulmonary artery resistance index is more accurate for prediction of neonatal respiratory distress syndrome in preterm fetuses: a pilot study. J Matern Fetal Neonatal Med. 2019 Feb;32[4]:626-632. DOI: 10.1080/14767058.2017.1387891.

27. White VA, Walker KF, Thornton JG. Trials of antenatal corticosteroids for preterm fetal lung maturity: a review of the potential for selective outcome reporting. Eur J Obstet Gynecol Reprod Biol. 2019 May;236:58-68. DOI: 10.1016/j.ejogrb. 2019.02.031.

28. Witlin AG, Saade GR, Mattar F, Sibai BM. Predictors of neonatal outcome in women with severe pre-eclampsia or eclampsia between 24 and 33 weeks' gestation. Am J Obstet Gynecol. 2000 Mar;182[3]:607-11. DOI: 10.1067/mob.2000. 104224.

29. Crowther CA, Middleton PF, Voysey M, Askie L, Zhang S, Martlow TK, et al. Effects of repeat prenatal corticosteroids given to women at risk of preterm birth: An individual participant data meta-analysis. PLoS Med. 2019 Apr 12;16[4]:e1002771. DOI: 10.1371/journal.pmed.1002771.

30. Weitzner O, Yagur Y, Weissbach T, Man El G, Biron-Shental T. Pre-eclampsia: risk factors and neonatal outcomes associated with early- versus late-onset diseases. J Matern Fetal Neonatal Med. 2020 Mar;33[5]:780-784. DOl: 10.1080/14767058.2018.1500551.

31. Eraslan Sahin M, Col Madendag I, Sahin E, Madendag Y, Acmaz G, Bastug O, Ozdemir A. Fetal Pulmonary Artery Acceleration/Ejection Ratio for Transient Tachypnea of the Newborn in Uncomplicated Term Small for Gestational Age Fetuses. Eur J Obstet Gynecol Reprod Biol. 2020 Apr;247:116120. DOI: 10.1016/j.ejogrb.2020.02.018. 


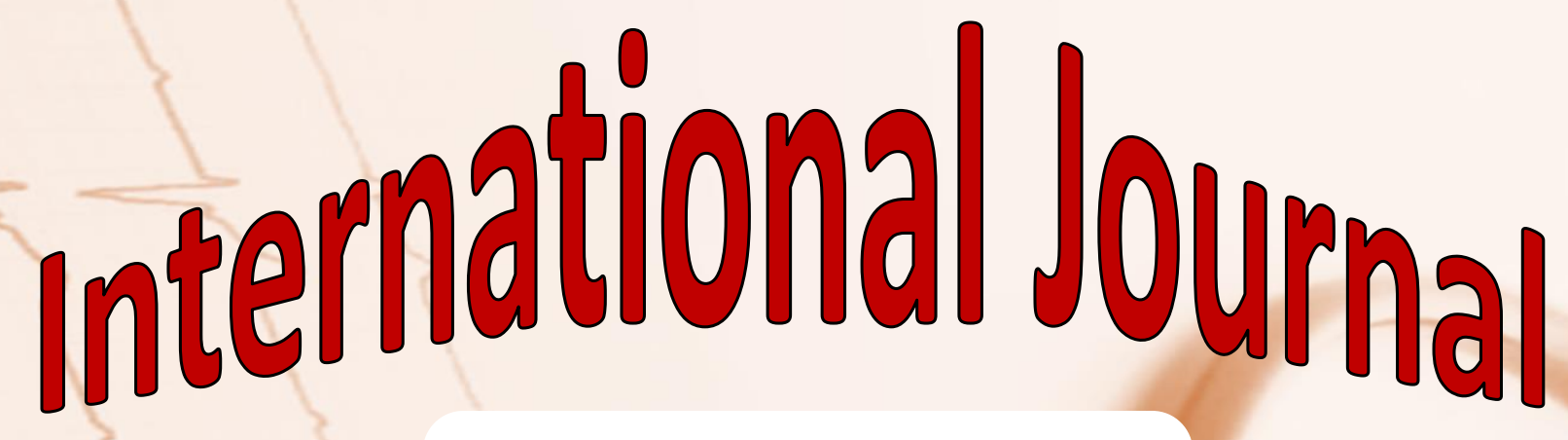

https://ijma.journals.ekb.eg/ Print ISSN: 2636-4174 Online ISSN: 2682-3780

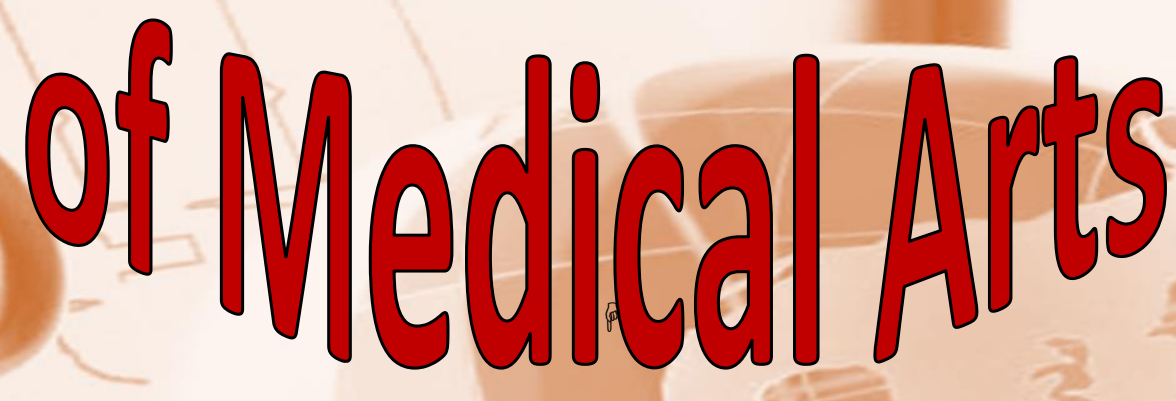

